\title{
Musculoskeletal disorders - disease burden and challenges in the developing world
}

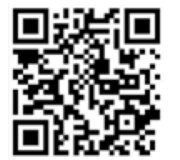

Recent advances in rheumatology have contributed to elucidate the complex pathogenic processes that underlie the development and progression of rheumatic diseases. This has led to the advent of new therapies to treat these conditions, including the biologic therapies. The use of synthetic disease-modifying anti-rheumatic drugs (DMARDs) together with biologic therapies has increased dramatically across a range of diseases, and resulted in improved outcomes for patients. In a study done in Spain, the overall age-standardised mortality rate for systemic lupus erythematosus (SLE) increased during 1981 - 1999 and stabilised during $2000-2010 .{ }^{[1]}$ The mean age at death increased with time, from 42 years in 1981 to 61 years in $2010 .{ }^{[1]}$ Similar mortality trends have been observed in patients with rheumatoid arthritis (RA), even though mortality still remains higher in RA patients compared with the general population. ${ }^{[2]}$ The overall SLE survival rates have increased significantly between the 1950s and 2000s, from $74.8 \%$ to $94.8 \%$ and $63.2 \%$ to $91.4 \%$ for the overall 5 -year and 10 -year survival, respectively. ${ }^{[3]}$ These improved outcomes are thought to be due to a combination of earlier recognition of mild disease and better approaches to therapy.

The European League Against Rheumatism (EULAR) and the American College of Rheumatology (ACR) have recently joined forces and contributed significantly to improved outcomes in rheumatic diseases by revising the classification criteria for most of these diseases, with the new criteria having higher sensitivity and specificity, which contribute to an early diagnosis. The latest contribution was the 2015 ACR/EULAR classification criteria for gout. ${ }^{[4]}$ Diagnostic imaging with magnetic resonance imaging (MRI) and ultrasonography has provided a new perspective to the management of arthritis, revealing subclinical inflammation and predicting progression of joint damage - thus contributing to early detection. ${ }^{[5]}$ Therefore, much more can be done for patients before the development of irreversible damage.

However, despite this improvement in outcomes, musculoskeletal (MSK) disorders still rank highest in prevalence as the cause of chronic ill-health, long-term disability, and consultation with healthcare professionals, as was shown in the Burden of Global Disease 2010 study ${ }^{[6]}$ This is against the background of MSK disorders contributing only $3.4 \%$ and $1.7 \%$ of the total disease burden in the developed and developing world, respectively. ${ }^{[6]}$ Osteoarthritis and low back pain remain the largest contributors to the global disability burden ${ }^{[6]}$ The Bone and Joint Decade 2000 2010, endorsed by many organisations including the World Health Organization (WHO) and many governments, has acknowledged the rising burden of MSK disorders. ${ }^{[7]}$ In 2013, the Global Burden of Disease (GBD) study showed that the proportion of disabilityadjusted life-years (DALYs) caused by years lost due to disability (YLD) increased globally from $21.1 \%$ in 1990 to $31.2 \%$ in $2013{ }^{[8]}$ Furthermore, the burden of MSK disorders in terms of DALYs in the developing world (21 076000 DALYs) is estimated to be almost 2.5 times that in the developed world (8 723000 DALYs). ${ }^{[6]}$
For SLE, survival rates in developing countries are significantly lower than those reported in developed countries such as the USA, Western Europe and Canada, where 5- and 10-year survival rates surpass $90 \% .{ }^{[9]}$ In South Africa (SA), the 5-year survival rate was shown to be between $57 \%$ and $72 \%(1986-2003)$ in a case series of 226 SLE patients. ${ }^{[9]}$ These studies show that MSK disorders are the largest contributors to disability and chronic ill-health, and further indicate the wide gap between developing and developed countries.

Developing countries are faced with several challenges in relation to reducing the burden of MSK disorders. In Africa, rheumatology as a specialty is considerably neglected and under-funded. It has to compete for scarce resources, which are allocated to communicable diseases such as tuberculosis, HIV and malaria, all of which are life threatening compared with the causes of disability. A systematic review carried out to address the prevalence of arthritis in Africa identified the paucity of prevalence data in Africa ${ }^{[10]}$ Funding for rheumatologyrelated research is inadequate and, in addition, the ratio of rheumatologists to population is high, in one study ranging from 1:35 000 to $1: 1600000 .^{[9]}$ Training of rheumatologists is insufficient and there is difficulty accessing them; this leads to delays in appropriate treatment and further contributes to disability, as the window of opportunity to start treatment has been missed. In SA, some provinces, such as Limpopo, do not have rheumatologists and some, such as the Eastern Cape, have one rheumatologist serving the entire population of about 6.9 million. ${ }^{[11]} \mathrm{In}$ developed countries, nurses are trained to deal with MSK disorders; they are key in managing these disorders. One other challenge is that of limited medication options due to unaffordability and/ or unavailability.

The major contributor to the GBD is the increase in the ageing population. As people become older, their likelihood of suffering from MSK disorders increases and quality of life deteriorates. To improve outcomes for MSK disorders in developing countries, there needs to be a change in emphasis, so that larger resources are allocated to non-communicable diseases. Medical schools have to introduce students to the diagnosis and treatment of MSK disorders early, general physicians and other specialists need to have greater exposure to these conditions and primary care workers should be trained to manage these disorders early with DMARD therapy. Resources should be allocated for the training of more rheumatologists and, more importantly, rheumatology nurses. CME could play a vital role in the dissemination of information to practitioners. Organisations such as the Arthritis Foundation of SA, a patient-orientated service, need to be established in other subSaharan countries and patients informed about new developments relating to MSK disorders. The use of MRI and ultrasonography has to be encouraged and funds have to be invested to provide these services in state-owned medical facilities. The 21st century hails a new era in the management of MSK disorders and it is hoped that the GBD from MSK disorders will decrease as people live longer. 


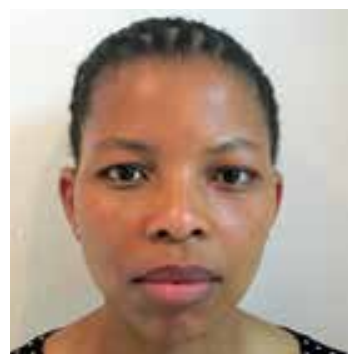

\section{Ayanda Gcelu}

Guest editor

ayanda.gcelu@uct.ac.za

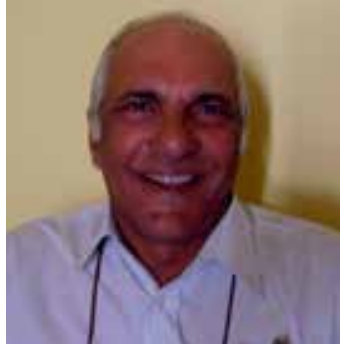

Asgar Ali Kalla

Guest editor

kallaasgar.kalla@uct.ac.za
1. Ruiz E, Ramalle-Gómara E, Elena Á, et al. Trends in systemic lupus erythematosus mortality in Spain from 1981 to 2010. Lupus 2014;23(4): 431-435. [http://dx.doi. org/10.1177/0961203313517015

2. Widdiefield J, Bernatsky S, Paterson JM, et al. Trends in excess mortality among patients with rheumatoid arthritis in Ontario, Canada. Arthritis Care Res (Hoboken) 2015;67(8):1047-1053. [http:// dx.doi.org/10.1002/acr.22553]

3. Mak A, Cheung MW, Chiew HJ, et al. Global trend of survival and damage of systemic lupus erythematosus: Meta-analysis and meta-regression of observational studies from the 1950s to 2000s. Semin Arthritis Rheum 2012;41(6):830-839. [http://dx.doi.org/10.1016/j. semarthrit.2011.11.002]

4. Neogi T, Jansen TLTA, Dalbeth N, et al. 2015 Gout Classification Criteria: An American College of Rheumatology/European League Against Rheumatism Collaborative Initiative. Arthritis Rheum 2015;67(10):2557-2568. [http://dx.doi.org/10.1002/art.39254]

5. Filippucci E, Geso LD, Grassi W. Progress in imaging in rheumatology. Nat Rev Rheumatol 2014;10:628-634. [http://dx.doi.org/10.1038/nrrheum.2014.145]

6. Adebajo A, Gabriel SE. Addressing musculoskeletal health inequity in Africa. Arthritis Care Res 2010;62(4):439-441. [http://dx.doi.org/10.1002/acr.20032]

7. Weinstein SL. 2000 - 2010: The Bone and Joint Decade. J Bone Joint Surg Am 2000;82(1):1-3.

8. Vos T, Barber RM, Bell B, et al. Global, regional, and national incidence, prevalence, and years lived with disability for 301 acute and chronic diseases and injuries in 188 countries, 1990 - 2013: A with disability for 301 acute and chronic diseases and injuries in 188 countries, 1990 - 2013: A
systematic analysis for the Global Burden of Disease Study 2013. Lancet 2015;22:386(9995):743-800. systematic analysis for the Global Burden of Disease Study 2013. Lancet 2015;22:386(9995):743-800. [http://dx.doi.org/10.1016/50140-6736(15)60692-4]

9. Wadee S, Tikly M, Hopley M. Causes and predictors of death in South Africans with systemic lupus erythematosus. Rheumatology (Oxford) 2007;46:1487-1491.

10. Usenbo A, Kramer V, Young T, et al. Prevalence of arthritis in Africa: A systematic review and metaanalysis. PLoS One 2015;10(8):e0133858. [http://dx.doi.org/10.1371/journal.pone.0133858]

11. Mid-year population estimates 2015. www.statssa.gov.za (accessed 12 November 2015).

S Afr Med J 2015;105(12):1070-1071. DOI:10.7196/SAMJ.2015.v105i12.10260 